\title{
Underactuated tendon-driven robotic/prosthetic hands: design issues
}

\author{
Annick Mottard, Thierry Laliberté and Clément Gosselin \\ Département de génie mécanique \\ Université Laval, Québec, Québec, Canada, G1V0A6 \\ Email: [thierry,gosselin]@gmc.ulaval.ca
}

\begin{abstract}
This paper discusses several design issues pertaining to the design of anthropomorphic underactuated robotic or prosthetic hands. The design of the underactuation mechanism that drives the fingers is first addressed. A double-stage mechanism is proposed that includes a lever arm to couple the motion of the thumb and the fingers as well as an additional pulley that amplifies the input force. Then, the design of the underactuated fingers is discussed and a novel rotational sliding joint is proposed. The geometric and mechanical design of a thumb mechanism is also presented that allows the reconfiguration of the thumb in the three most favorable poses. Finally, a prototype is presented that implements the design choices discussed in the paper and its effectiveness is qualitatively discussed based on a series of experiments.
\end{abstract}

\section{INTRODUCTION}

The design of underactuated robotic and prosthetic hands has been addressed in the literature for several decades. The concept of underactuation in prosthetic hands can in fact be traced back to the nineteenth century (see for instance [21]). In robotics, the large number of degrees of freedom in a multi-fingered hand poses a great challenge in terms of design (large number of actuators, complex transmissions) and control (coordination of the degrees of freedom in the performance of a task). This has motivated the use of underactuation, i.e., the introduction of mechanisms that distribute the action of a certain number of actuators to a larger number of degrees of freedom. This concept is sometimes referred to as mechanical intelligence [22] because part of the grasping intelligence is embedded in the mechanics of the hand. Early designs of robotic underactuated hands (see for instance [3, 15]) made use of either linkages or tendon/pulley systems to implement this concept. The mechanical analysis of such underactuated robotic systems has then been formalized (e.g. in [5]).

One of the critical building blocks required to implement underactuation in robotic hands is the design of underactuated fingers. This topic has been addressed in many papers and patents and is considered a somewhat mature topic. Examples of sucessful finger designs based on linkages or tendons can be found in [18, 19, 6]. Nevertheless, the integration of underactuated fingers into an effective hand that includes underactuation between the fingers remains a complex design and control problem. One approach that was proposed for the coordinated control of several degrees of freedom is that of hand synergies, which is inspired from the functionality of the human hand (see for instance [7, 14]).
In prosthetics and in some robotic applications, anthropomorphic hands are a desirable concept. Moreover, in prosthetics, there is a strong incentive to reduce the number of active inputs to only one, because of the difficulty to obtain more than one control signal (e.g. electromyographic signals) or driving input (e.g. shoulder harness and cable mechanical input). Therefore, several designs with a single input have been proposed in the literature (see for instance [8, 17, 12]).

This paper addresses some of the issues involved in the design of a tendon-driven anthropomorphic underactuated hand with a single actuation input. Instead of presenting a detailed description of the design of the prototype that was built, the paper is structured around design issues, in order to provide insight to the reader. First, the force transmission characteristics of the mechanism that drives the motion of the fingers is discussed. Then, the design of the fingers themselves is addressed, mainly the number and types of joints to be used. A novel rotational sliding joint that provides robustness to abduction/adduction forces is proposed. The geometric design of the thumb is then discussed, including means of producing a set of useful orientations of the thumb with respect to the palm and other fingers using a single revolute joint at the base of the thumb. The mechanism proposed to lock the thumb in each of these configurations is also presented and the experimental validation of a prototype is then briefly illustrated. Finally, a discussion is proposed to provide insight on the design issues addressed in the paper and to comment on the advantages and drawbacks of the prototype.

\section{FORCE TRANSMISSION}

The possibility to operate an underactuated hand using a single actuator is attractive, especially in prosthetic applications, where minimizing the weight is of paramount importance. Although this approach is less critical in robotics applications, it is nevertheless of great interest to minimize the number of control inputs in order to simplify the task planning operations.

The distribution of an input force on several output links has been addressed in many papers, especially in the context of underactuated hands (see for instance [20, 18]). In tendondriven hands, pulleys, sliders and intermediate bodies can be used to this end. However, such transmissions must be carefully designed in order to avoid introducing unwanted 


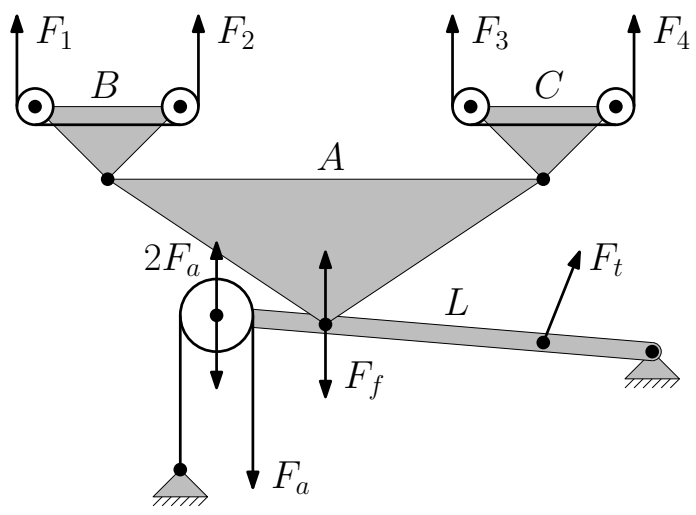

Fig. 1. Schematic of the transmission mechanism, including the input pulley that amplifies the force, the lever arm that couples the motion of the thumb with that of the fingers and the double-stage underactuation mechanism.

friction, which can significantly reduce the effectiveness of the hand and lead to poor performances. For example, sliders with linear guiding rails should be avoided since they introduce friction and are prone to jamming. In [1], several pulley and floating-body arrangements are studied for their force transmission qualities. A combination of some of these arrangements has been used in [2] with good results. One limitation noted by the authors was the lack of grasping force for a given actuation force. Therefore, the arrangement is slightly modified here in order to further increase the force transmission ratio. Mainly, the input actuation force $F_{a}$ is doubled using an alternative cable routing, as illustrated in Fig. 1. The remainder of the mechanism is similar to the one presented in [2]. The motion of the fingers and the thumb are coupled to the actuation motion through a transmission lever $L$. The motion of the thumb is coupled to the motion of the other fingers while preserving the shape adaptation capability because they work in opposition (the adaptation is naturally performed by the relative motion between the object and the hand during the closing phase). Moreover, this coupling allows the relative position of the opposing fingers during actuation to be predicted, which is especially useful to achieve pinch grasps. The lever provides a means of adjusting the distribution of the forces/velocities between the thumb and the other fingers. In order to obtain a correct behaviour, the closing speed of the thumb should be smaller than that of the fingers. The lever allows this feature if the input of the thumb $F_{t}$ is located closer to the fixed pivot than the input of the fingers $F_{f}$ (see Fig. 1). It is noted that the distribution of the forces between $F_{f}$ and $F_{t}$ depends on the shape of the object to be grasped and on the disturbances applied on it.

Then, an underactuation mechanism allows adaptation of the four fingers to the shape of the object and distributes approximately equally the force $F_{f}$ among the four fingers with forces $F_{i}$ (see Fig. 1). A first stage $A$ distributes the force to the two units $B$ and $C$ of the second stage. Because of its simplicity, a see-saw mechanism is used for the first stage. Also, because the see-saw mechanism is large, the tilt angle is sufficient to adapt to the closing variations of the fingers.
For the second stage, floating-pulley mechanisms are used because the circulation of the cable on the pulleys allows a large range of adaptation to the closing variations of the fingers (the tilt angle of a small see-saw mechanisms would not be sufficient). Moreover, the floating-pulley mechanisms interface with the tendons of the fingers without any attachment. Indeed, only one cable is used for two fingers: it is attached at the tip of a first finger, then goes through that finger, through the floating-pulley mechanism, to the other finger and is finally attached at the tip of the other finger. Globally, the underactuation mechanism induces very little friction and produces large output forces for a given input actuator force.

\section{FINGER DESIGN}

\section{A. Number and type of joints}

Several underactuated hands proposed in the literature include three phalanges per finger. This choice follows naturally from anthropomorphism and is also motivated by the desire to ensure that the fingers can adapt to the shape of arbitrary objects. Along this line, some designs include even larger numbers of phalanges like for instance the pioneer design presented in [15]. Nevertheless, as shown in [4], for a given input force, the contact force tends to decrease relatively rapidly from one phalanx to the next due, among other reasons, to the necessity to preserve the stability of the finger and to the unavoidable friction in the transmission. Moreover, it can be observed that the range of motion of the last interphalangeal joint of the human finger is much smaller than that of the middle joint. Therefore, limiting the number of phalanges to two by removing the distal interphalangeal joint — joint connecting the second phalanx to the third - is a possible option. The distal interphalangeal joint can be replaced by a fixed bend in the second phalanx. The length of the second phalanx is also extended to include the length of the second and third phalanges. The angle of the bend included in the second phalanx is a compromise between the ability to grasp small objects and the stability of grasps for large objects. According to [16], an angle of $20^{\circ}$ is a good compromise in this context.

Another issue in the design of the fingers is the type of joints to be used. Most robotic hand designs use revolute joints. Revolute joints are very common in mechanical systems. They are easy to design and conceptually simple. However, in robotic fingers, where successive phalanges must be aligned without offsets, revolute joints limit the range of motion of the joints, which has a significant impact on the capabilities of fingers to envelop small objects. Therefore, other types of joint are desirable and have been used in some hand designs. For example, each joint of the fingers of the anatomically correct testbed hand (ACT) proposed in [10] consists of a combination of two revolute joints geometrically arranged to replicate the kinematics of human fingers. However, the design of such joints is rather complex. Other designs use rolling joints, which provide large ranges of motion. The challenge in the design of rolling joints is to ensure that contact is maintained between the rolling surfaces and to avoid slippage between the rolling 
components. In [7], gears are used to implement rolling joints, which eliminates slippage and provides proper transmission characteristics.

In this work, another type of joint is proposed for the fingers, namely rotational sliding joints. The geometry of these joints is inspired by the human anatomy, namely the shape of the bones. In these joints, the end of the link closest to the base of the fingers is shaped as a convex cylinder with a rounded slot while the distal link is shaped as a concave cylinder that fits in the slot of the proximal link. The links are maintained in contact using elastic belts and the two links can slide on one another. This is illustrated in Fig. 2 while the tendon routing is shown in Fig. 3. The tendon that goes through the pulleys and that is attached near the tip of the finger is the stiff actuation tendon. The tendon located near the back of the finger is an elastic cable that produces the return action, i.e., the opening of the finger when the actuation forces are released. The finger in its fully closed configuration is shown in Fig. 4 The unloaded closing sequence of the fingers (no contact with objects) is determined by the actuation lever arms, the stiffness of the elastic return cable, the mechanical limits, the pulley diameters and the friction forces. The elastic belts at the joints ensure that the finger joints return to their minimum energy configuration after being subjected to abduction/adduction forces, as well as torsional or axial loads (phalanges pulled apart). It can be noted that the basic principle of the rotational sliding joints involves friction between consecutive phalanges. However, the pressure distribution on the surfaces in contact and the small ratio of the lever arm of the friction forces over the lever arm of the external forces on the finger make this friction acceptable. Compared to geared joints, the sliding joints are simpler. Also, they provide very good stability and robustness to external forces and moments, as described in the next subsection.

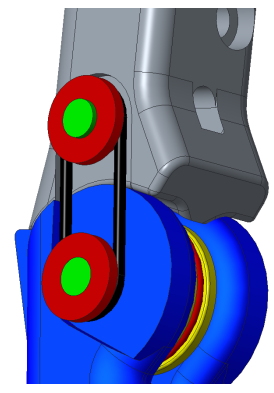

(a) Design of the joints.

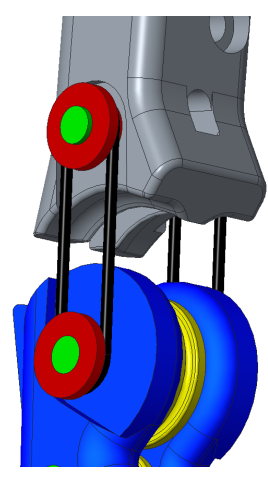

(b) Joint pulled apart.
Fig. 2. Rotational sliding joint.

\section{B. Robustness to abduction/adduction forces}

Another significant advantage of rolling or rotational sliding joints making use of elastic components to maintain the surfaces in contact is their robustness to external forces, as demonstrated in [7]. This feature also includes robustness to abduction/adduction forces, which can occur in the case of

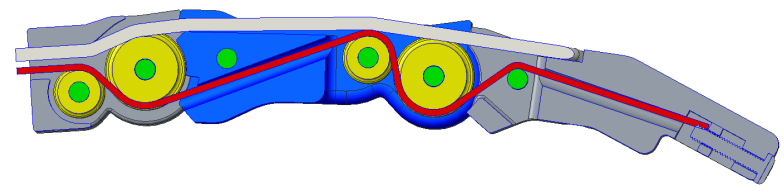

Fig. 3. Routing of the actuation and return tendons.

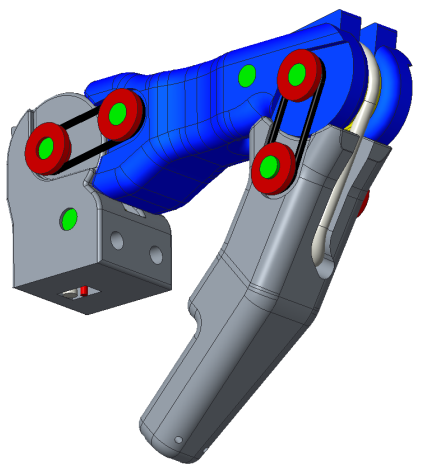

Fig. 4. Finger in fully closed configuration.

unwanted collisions or other impacts. The arrangement of the joints that allows the passive abduction/adduction motion is also inspired from the human anatomy. As it can be observed in [13], the intrinsic palm muscles (dorsal interossei), that act to abduct (spread) the index, middle, and ring fingers away from the hand's midline, can also provide compliance in the case of lateral shocks. The soft tissues located on the sides of the hand also help in that respect. The elastic belts that hold the rolling or rotational sliding joints together in robotic hands yield a similar behaviour.

In the hand design proposed in this work, this arrangement provides a compliance that protects the hand and the objects from impacts, as illustrated in Fig. 5. Moreover, when lateral forces are applied at the tip of the fingers, since the lever arm to the metacarpophalangeal joint (closest to the palm) is longer, the moment at this joint is the largest and the fingers tend to pivot laterally around this joint (and not around the interphalangeal joint(s)), which is the desired behaviour. This appears clearly in Fig. 5 Nevertheless, a deformation at the interphalangeal joint is also possible if the contact situation requires it, which is an additional means of protecting the components. The possible elastic deformation of the hand makes it very robust to external impacts. Additionally, in the event of a failure, the elastic belts that hold the joints together act as a mechanical fuse. Indeed, failure is much more likely to occur at the elastic belts than in the links or joints themselves. Since the elastic belts are easily accessible - they are mounted on the sides of the fingers - and since they are easy to replace, recovery from a failure is not a difficult task. This 'softness' of the hand does not compromise its grasping force capability. However, a possible drawback of the compliance of the joints relative to abduction/adduction motions is that the fingers may sag laterally when heavy 


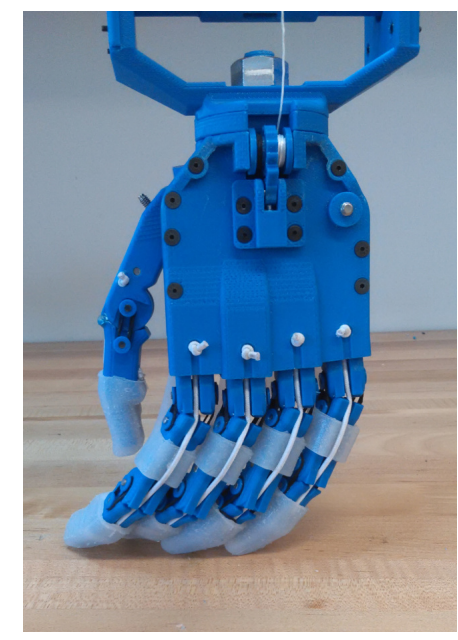

Fig. 5. Lateral deformation of the hand.

objects are grasped (for instance grasping a heavy bottle that is held vertically). Therefore, a compromise should be reached between compliance and lateral force capability.

\section{THUMB DESIGN AND GEOMETRIC CONFIGURATION}

Depending on the type of grasp to be performed (see for instance the taxonomies provided in [9, 11]) the position and orientation of the thumb relative to the other fingers must be adapted. This is a challenge for underactuated anthropomorphic hands in which a single actuation input is used. Indeed, additional mechanisms or locking features must be included in the design in order to allow the thumb to be stably reconfigured. It is noted that this challenge also exists in nonanthropomorphic hands, although it is not as critical (see for instance the reconfiguration strategies used in [18]).

In an anthropomorphic hand, three main configurations can be identified for the thumb, namely: $i$ ) to perform spherical or precision grasps, the thumb should oppose the index and middle finger in order to ensure the stability of the grasps, ii) to perform cylindrical grasps, the thumb should be offset from the other fingers in order to allow a complete closure on the object without mechanical interference of the fingers and iii) to perform lateral grasps, the thumb should be in an abducted configuration (thumb coplanar with the other fingers). In this last case, the closing motion of the thumb should be essentially parallel to the plane of the palm. In a prosthetic hand, the reconfiguration of the thumb can be performed externally, by the other hand of the user. In a robotic hand, reconfiguration may be accomplished by extra actuation channels or by pushing the thumb against external surfaces using the robot on which the hand is mounted. In this work, the assumption is made that external means of reorienting the thumb are available, like in prosthetics. Therefore, the main issue is the design of the reconfiguration mechanism itself. The motion undergone by the thumb around its base is rather complex. However, it is desirable to simplify this motion in order to reduce the complexity of the required mechanical design. To this end, the three configurations described above for the thumb are analysed in order to determine a unique rotation axis that can produce the three relative poses of the thumb that correspond to these configurations. The analysis is performed using the geometric description provided in Figs. 6 and 7. where configuration $A$ corresponds to case $(i)$ above (opposition with index and middle finger) while configuration $B$ corresponds to case (iii) above (thumb abducted). The intermediate configuration $(i i)$ is verified a posteriori. The precise specification of configurations $A$ and $B$ is somewhat heuristically obtained, although it is strongly guided by the grasps associated with these configurations. Point $P$, the origin of the palm-fixed reference frame, is placed at the base of the thumb. It is placed rather low on the palm in order to obtain an anthropomorphic design. Based on the above model, it is now required to determine the fixed rotation axis through $P$ that allows frame $B\left(x_{b}, y_{b}, z_{b}\right)$ to be moved to frame $A\left(x_{a}, y_{a}, z_{a}\right)$. One can then write

$$
\mathbf{Q}_{b}=\mathbf{Q}_{r} \mathbf{Q}_{a}
$$

where $\mathbf{Q}_{b}$ is the rotation matrix from the fixed frame $P$ to frame $B, \mathbf{Q}_{a}$ is the rotation matrix from the fixed frame $P$ to frame $A$ and $\mathbf{Q}_{r}$ is the rotation matrix from frame $A$ to frame $B$ and where all rotation matrices are expressed in the fixed frame $P$ (hence the order of the matrix product). Equation 1 is easily rewritten as

$$
\mathbf{Q}_{r}=\mathbf{Q}_{b} \mathbf{Q}_{a}^{T}
$$

and from Fig. 7, one can also write

$$
\mathbf{Q}_{a}=\left[\begin{array}{ccc}
\cos \beta \cos \phi & -\cos \beta \sin \phi & \sin \beta \\
\sin \phi & \cos \phi & 0 \\
-\sin \beta \cos \phi & \sin \beta \sin \phi & \cos \beta
\end{array}\right]
$$

and

$$
\mathbf{Q}_{b}=\left[\begin{array}{ccc}
\cos \alpha & -\sin \alpha & 0 \\
\sin \alpha & \cos \alpha & 0 \\
0 & 0 & 1
\end{array}\right]
$$

where angle $\beta$ is equal to $\pi / 2$, i.e., the rotation from the opposing configuration to the lateral configuration. Angles $\alpha$ and $\phi$ are empirically chosen to mimic the human hand and to produce the required grasping configurations. Finally, the axis of rotation, given by unit vector $\mathbf{v}_{r}$, is determined using

$$
\mathbf{v}_{r}=\frac{\operatorname{vect}\left(\mathbf{Q}_{r}\right)}{\left\|\operatorname{vect}\left(\mathbf{Q}_{r}\right)\right\|}
$$

where vect $(\cdot)$ stands for the vector linear invariant of its matrix argument and $\|\cdot\|$ stands for the Euclidean norm of its vector argument. In other words, unit vector $\mathbf{v}_{r}$ is the eigenvector of matrix $\mathbf{Q}_{r}$ associated with the eigenvalue 1 .

Obviously, the solution provided by the above equations is dependent on the values chosen for $\alpha$ and $\phi$. The following considerations on configuration $A$ are taken into account in the choice of a design solution, namely: $i$ ) the thumb must be far enough from the palm, $i i$ ) the interphalangeal joint of the thumb is slightly lower than the metacarpophalangeal joints of the fingers and iii) the thumb is closing perpendicularly to the palm. Similarly, the following considerations on configuration $B$ are taken into account: $i$ ) the interphalangeal joint of the 


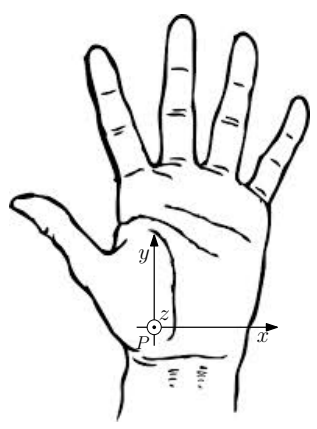

Fig. 6. Palm-fixed reference frame.

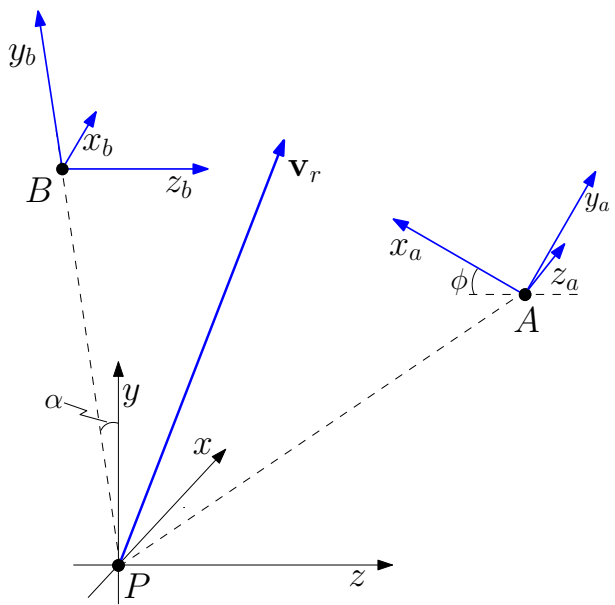

Fig. 7. Initial and final configurations of the thumb with unique rotation axis.

thumb is approximately at the same height as the metacarpophalangeal joints of the fingers, $i i$ ) the thumb is relatively close to the index, while leaving a large enough gap to grasp an object and iii) the thumb is closing in a plane parallel to that of the palm. These considerations still leave the designer with a relatively large design space, which can be useful since many other practical constraints may exist. In this work, the solution chosen yields

$$
\mathbf{v}_{r}=[0.147,0.978,0.147]^{T} .
$$

Referring to Fig. 8, this corresponds to an inclination of the base rotation axis of the thumb of $\psi=8.5^{\circ}$ and $\theta=81.5^{\circ}$. The resulting design of the base of the thumb is shown in Fig. 9

One additional issue with the reconfiguration of the thumb is the possible mechanical interaction between the motion of the thumb associated with the reconfiguration and the closing motion of the thumb. In some designs (see for instance [2]), the tendon actuating the closing motion of the thumb passes through the base pivot of the thumb, which guarantees that the closing action of the tendon does not affect the configuration of the thumb. However, in the design presented in this paper, the tendon does not pass through the base axis, which means that if the thumb is not stabilized, the closing action of the tendon will tend to move the thumb to the configuration associated

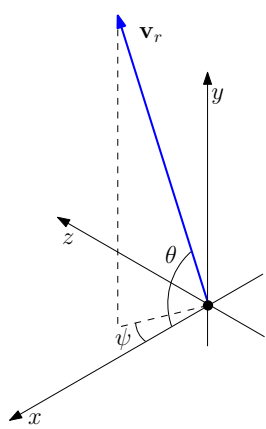

Fig. 8. Orientation of the thumb rotation axis.

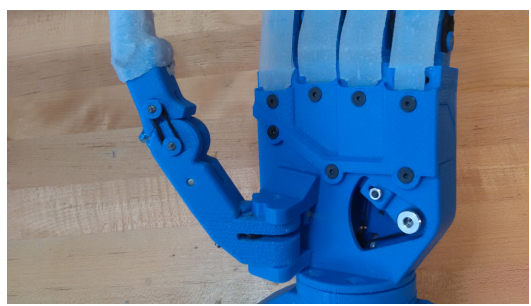

Fig. 9. Revolute joint at the base of the thumb.

with spherical grasps (thumb opposing the fingers). Therefore, a cam system is included at the base of the thumb in order to stabilize its configuration. It is noted that such a system may be desirable even in situations in which the actuation tendon of the thumb passes through its pivot axis, in order to improve stability against external forces. For this reason, although a thumb that would not include a cam would potentially allow infinitely many configurations, it is not desirable since its motion could be unstable. The cam system proposed here is shown in Fig. 10 In this design, the follower is connected to a pulley in the proximal link of the thumb. The follower is also mounted on a spring, which keeps it in contact with the cam. The profile of the cam includes three smooth indentations in which the follower can engage to lock the thumb in one of the three configurations (see Fig. 10). Additionally, with this arrangement, when the tendon is pulled to close the thumb, the resulting forces tend to maintain the follower in the indentation of the cam. In other words, the thumb configuration is very stable when the grasping action is taking place but it is easy to reconfigure when no grasping force is applied, which is a desirable behaviour in prosthetics as well as in robotics applications. Since the position of the pulley changes with the configuration of the thumb, the minimal tension in the tendon going to the thumb also changes, which dictates the timing of the closing motion of the thumb with respect to the closing motion of the fingers. For instance, in the spherical grasp, the tension in the cable going to the thumb is smaller. Hence, the fingers first close towards the thumb while the thumb closes towards the fingers near the end of the grasp. On the other hand, in the lateral grasp, the tension in the cable going to the thumb is larger. In this case, the thumb first closes towards the index finger before the fingers are completely closed. Since the fingers and the thumb are mechanically coupled, almost all 

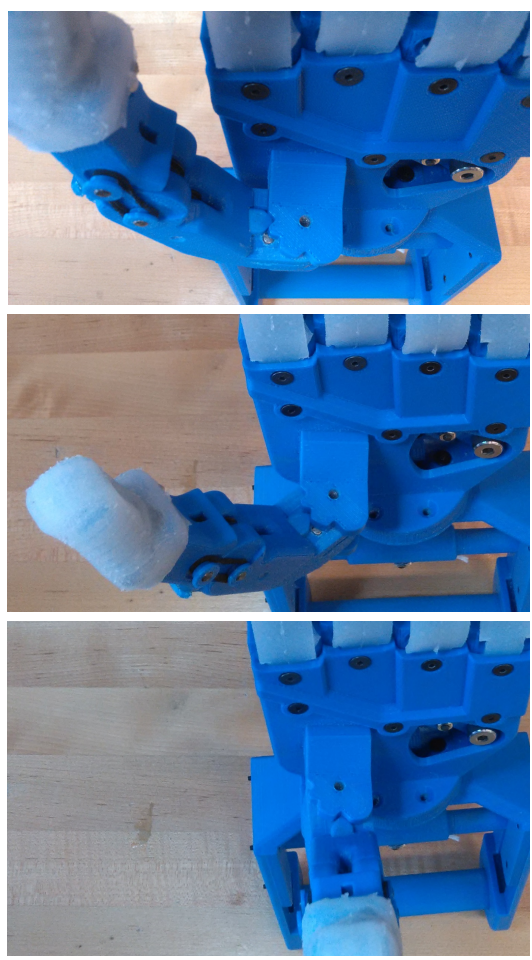

Fig. 10. Three positions of the cam mechanism and corresponding configurations of the thumb.

the actuation force is directed towards the thumb in this case (the fingers are not completely closed), which is the desired behaviour.

\section{LOCKING MECHANISM}

It can be tiresome for a user of a hand prosthesis to hold an object for a long period of time. Moreover, if another task is performed while holding the object, an involuntary motion can make the user drop the object. Therefore, there is an interest for a mechanism that maintains the hand closed. While locking mechanisms exist for some prostheses, a locking mechanism adapted for an underactuated hand prosthesis was not found in the literature.

Therefore, a specific locking mechanism, illustrated in Fig. 11, was developed. This mechanism, which allows to keep the hand closed by locking the input cable of the hand, is located at the back of the hand, close to the base. The input cable is wrapped and attached to a pulley that includes a ratchet. Then, a pawl can engage the ratchet and lock it if desired. A spring plunger drives the pawl in one of two configurations (similarly to the mechanism of a light switch). Also, the desired configuration can be selected manually using a lever attached to the pawl. If the locking mechanism is not needed, the pawl is disengaged. If the locking mechanism is desired, the pawl is engaged and the hand closes until the object is properly grasped (during the closing, a clicking noise confirms that the hand is in the locked mode). The grasp can then be maintained properly without effort. It is noted that because of the ratchet-pawl mechanism, the locking works for

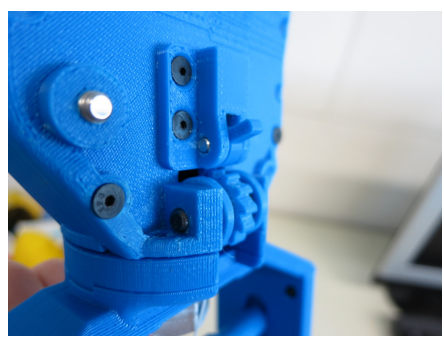

Fig. 11. Locking mechanism with ratchet and pawl.

objects of any size. When the user wants to release the object, the pawl is disengaged and the hand can open. It is noted that the end of the input cable attached to the lever and the end of the input cable attached to the actuator can be wrapped and attached to different sections of the same pulley. If these sections have different diameters, the pulley can be used to modify the transmission ratio.

\section{PRototy Ping AND EXPERIMENTAL ASSESSMENT}

The above described issues and design choices led to the design of a prototype of an anthropomorphic robotic/prosthetic hand. The different components were built using 3D printing except for shafts, screws and similar components. Parts were designed so that the prototype would be simple to assemble. This modular approach made it easy to test each of the components and subsystems individually and to make changes to the prototype. The total mass of the prototype is $298 \mathrm{~g}$ (without actuator). A handle was designed and mounted at the base of the hand such that the opening/closing motion of the hand can be operated manually. Using the manual operation of the hand, it is easy to quickly try a variety of grasps and grasping conditions. The configuration of the thumb is changed manually in order to pre-shape the hand for a given type of grasp. The reconfiguration of the thumb on the prototype is easy to perform and the feedback obtained from the indentations in the cam is clear and sharp. Soft finger pads were designed that cover areas as large as possible on the phalanges while providing compliance and high friction coefficient. A series of grasping tasks were conducted with the hand in order to assess its capabilities. Example results are now briefly described.

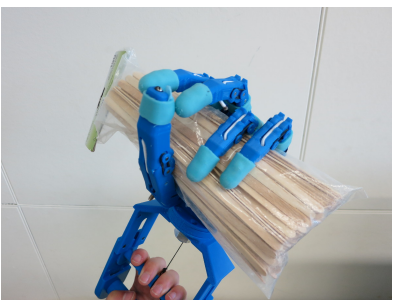

(a) Thumb opposing the fingers.

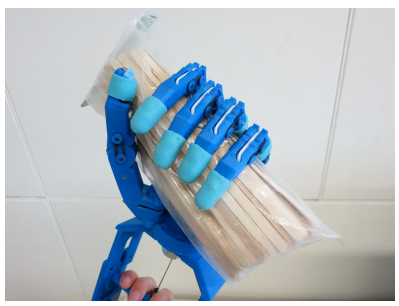

(b) Thumb in the intermediate configuration.
Fig. 12. Grasping of a cylindrical object.

In Fig. 12, one same object is grasped with two different 
strategies, namely two configurations of the thumb. The first picture shows the grasp that makes use of the spherical configuration (thumb facing the palm) while the second picture shows the grasp performed using the second configuration of the thumb (for cylindrical grasps). In the first case, it can be observed that the thumb makes contact with the index and with the middle finger, which prevents the fingers from enveloping the object completely. In the second case, the index and the middle finger can better envelop the object because the thumb is no longer interfering. This example shows the relevance of the intermediate configuration of the thumb. Indeed, smaller objects can be stably grasped using this configuration of the thumb, as shown in Fig. 13, where objects used in the activities of daily living are grasped (dust pan and screwdriver).

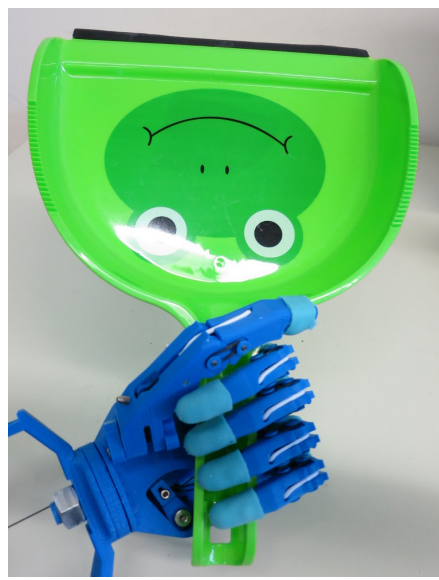

(a) Grasping a dust pan.

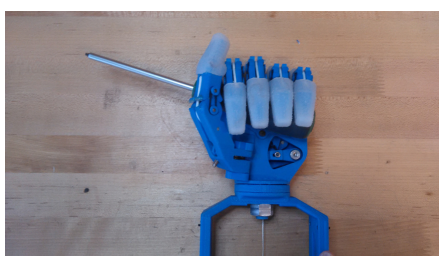

(b) Grasping a screwdriver.

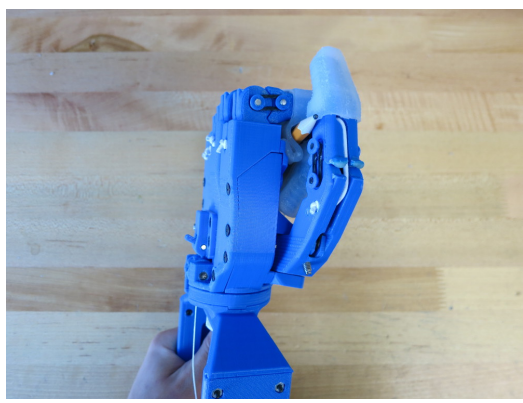

Fig. 14. Stable grasping of a pencil.

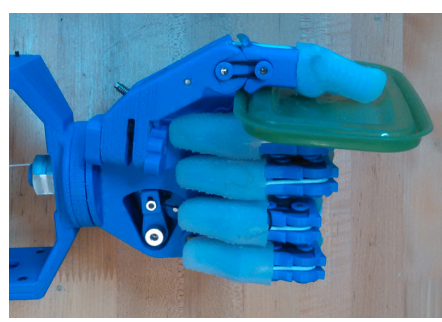

Fig. 15. Lateral grasp of a lid.

working in hyperextension in order to accommodate the large size of some of the objects.

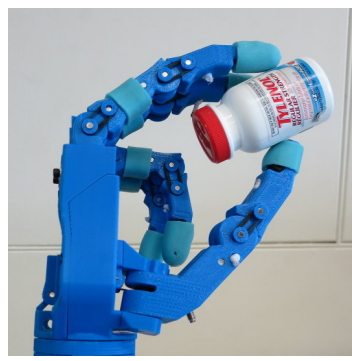

Fig. 16. Precision grasp using the spherical configuration.

Fig. 13. Grasping commonly used objects using the cylindrical grasp.

In order to grasp even smaller objects, the abducted configuration of the thumb (for lateral grasps) can be used. Figure 14 shows the hand grasping a pencil. It is noted that the soft covering of the fingers greatly helps with the grasping of small objects by filling the gap between the fingers and the palm.

The abducted configuration of the thumb can also be used to perform lateral grasps of thin objects, for which it was designed. Figure 15 shows this type of grasp.

The spherical configuration of the thumb (opposition with the index and middle finger) can be used to perform precision grasps, as shown in Fig. 16. Enveloping grasps can also be performed using the spherical configuration of the thumb. Figure 17 shows examples of such grasps. The enveloping grasps are very stable due to the underactuation between the fingers, which allows the hand to adapt to the shape of the object. Also, it can be observed in Fig. 17 that the thumb is

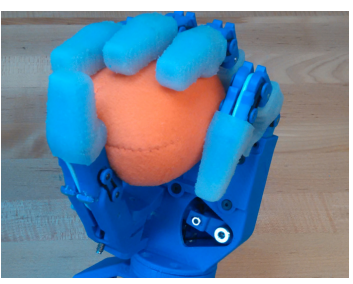

(a) Grasping a ball.

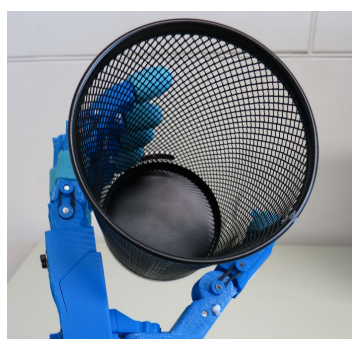

(b) Grasping a basket.
Fig. 17. Grasping larger objects using the spherical configuration.

Finally, the possible lateral elastic deformation of the fingers (abduction/adduction) is illustrated in Fig. 18. This feature protects the fingers in case of impacts. It also allows the fingers to further adapt to the shape of the grasped objects. 


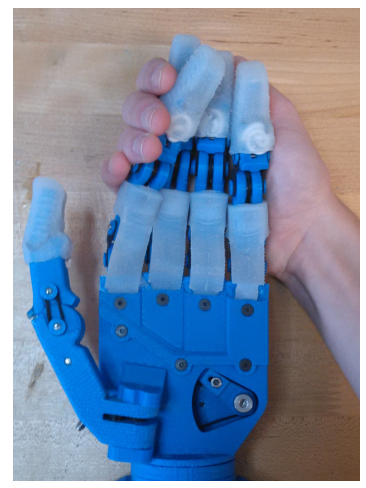

(a) Adduction of the fingers.

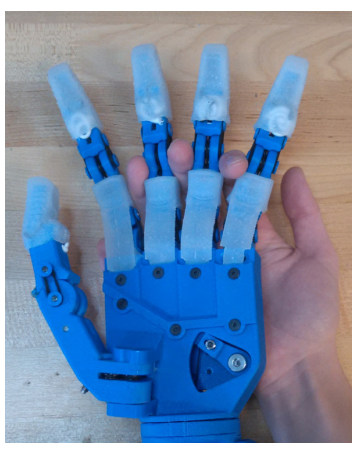

(b) Abduction of the fingers.
Fig. 18. Possible deformation of the fingers.

\section{DISCUSSION}

The experiments performed with the prototype reveal some advantages and drawbacks of the design choices made based on the discussions presented in this paper. First, the rotational sliding joints maintained in contact by elastic belts perform well and allow the fingers to deform under the action of lateral forces. As opposed to other designs based on rolling or gear joints found in the literature, the joints proposed here involve the sliding of the adjacent links on a cylindrical surface. The main advantage of this design is its simplicity of manufacturing and assembly. However, its drawback is that it introduces more friction than rolling or geared joints. In the prototype presented in this paper, although it was not a major issue, the effect of this friction was noticed (moderate increase of the actuation force compared with standard revolute joints). One possible avenue to reduce this friction is to reduce the radius of the rotational joints such that the friction forces are applied closer to the centre of the joint, thereby reducing the resulting moment. Another possible means of reducing the friction would be to include only one rotational sliding joint per finger (at the base of the finger) while using a revolute joint at the interphalangeal joint. Such an arrangement would still protect the finger from impacts and may be capable of producing the required ranges of motion. Another design choice that was made in this work was to use two phalanges in each of the fingers instead of three. The experiments demonstrated that this choice had very little impact on the capabilities of the hand while simplifying the design and improving the force transmission. In fact, using only two phalanges is believed to be one of the reasons why the rotational sliding joints led to very good force transmission characteristics despite the additional friction.

The design of the underactuation mechanism based on tendons, pulleys and see-saw mechanisms was also addressed. This component of the hand is critical to the transmission of the forces and it was tested independently before assembling the complete hand. Although the results of these tests are not reported here because of space limitation, it can be said that the underactuation mechanism yields excellent transmission ratios and introduces little friction due to its floating nature.
Additionally, the use of a pulley to double the force transmitted to the fingers for a given input force provides a significant advantage over direct drive designs. Finally, it should be noted that there is a direct mechanical connection between the thumb and the main actuation (the thumb and the fingers are not underactuated with respect to one another). This is justified by the fact that the thumb works in opposition with the other fingers. This actuation scheme proved to be very effective for all types of grasps performed with the hand. However, the routing of the tendon going from the actuation lever to the thumb in the current prototype should be simplified in order to further enhance this feature.

The geometric arrangement of the thumb is another critical issue in the design of an anthropomorphic underactuated hand. A kinematic model of the motion of the thumb was used to determine a unique rotation axis that produces the most favorable poses of the thumb with respect to the palm and other fingers. Although the model does not yield a unique solution, it provides a design space within which the geometric parameters can be chosen. Moreover, a cam system with indentations was proposed to lock the thumb in the orientations that correspond to the most common grasping configurations. The experiments conducted with the prototype confirmed that it is easy to switch from one configuration to another in a no-load situation while the configurations are very stably maintained when the grasping action is engaged. Finally, the novel locking mechanism provides the capability to effortlessly maintain a grasp, which can be very useful in practice.

\section{CONCLUSION}

Some of the issues pertaining to the design of single-input anthropomorphic tendon-driven underactuated hands were addressed in this paper, namely: force transmission, finger and joint design and thumb configurations and mechanisms. Each of these issues was discussed and design choices were made that led to a functional prototype that was tested experimentally. Qualitative results were presented and discussed. One of the main contributions of this work is the concept of rotational sliding joints, which are similar to rolling or geared joints but which are very simple to manufacture, assemble and operate. Other contributions are the design of the underactuated mechanism that drives the fingers, the thumb reconfiguration mechanism and the locking mechanism. The prototype of an anthropomorphic underactuated tendon-driven hand built in this work is also a contribution in itself. Its effectiveness was demonstrated by a series of qualitative tests briefly summarized in the paper.

\section{ACKNOWLEDGMENTS}

The financial support of the Natural Sciences and Engineering Research Council of Canada (NSERC) and of the Canada Research Chair Programme is gratefully acknowledged. The authors would also like to thank Mr. Jean-Michel Boisclair for his help with the preparation of the Computer-Aided Design (CAD) models of the hand. 


\section{REFERENCES}

[1] Mathieu Baril, Thierry Laliberté, François Guay, and Clément Gosselin. Static analysis of singleinput/multiple-output tendon-driven underactuated mechanisms for robotic hands. In ASME International Design Engineering Technical Conferences and Computers and Information in Engineering Conference, pages 155-164, 2010.

[2] Mathieu Baril, Thierry Laliberté, Clément Gosselin, and François Routhier. On the design of a mechanically programmable underactuated anthropomorphic prosthetic gripper. ASME Journal of Mechanical Design, 135(12): 121008, 2013.

[3] George A Bekey, Rajko Tomovic, and Ilija Zeljkovic. Control architecture for the Belgrade/USC hand. In Dextrous robot hands, pages 136-149. Springer, 1990.

[4] Lionel Birglen and Clément Gosselin. Kinetostatic analysis of underactuated fingers. IEEE Transactions on Robotics and Automation, 20(2):211-221, 2004.

[5] Lionel Birglen, Thierry Laliberté, and Clément Gosselin. Underactuated Robotic Hands. Springer, 2007.

[6] Gianni Borghesan, Gianluca Palli, and Claudio Melchiorri. Design of tendon-driven robotic fingers: Modeling and control issues. In IEEE International Conference on Robotics and Automation (ICRA), pages 793-798, 2010.

[7] Manuel G Catalano, Giorgio Grioli, Edoardo Farnioli, Alessandro Serio, Cristina Piazza, and Antonio Bicchi. Adaptive synergies for the design and control of the Pisa/IIT softhand. The International Journal of Robotics Research, 33(5):768-782, 2014.

[8] Jill D Crisman, Chaitanya Kanojia, and Ibrahim Zeid. Graspar: A flexible, easily controllable robotic hand. IEEE Robotics and Automation Magazine, 3(2):32-38, 1996.

[9] Mark R Cutkosky. On grasp choice, grasp models, and the design of hands for manufacturing tasks. IEEE Transactions on Robotics and Automation, 5(3):269-279, 1989.

[10] Ashish D Deshpande, Zhe Xu, Michael J Vande Weghe, Benjamin H Brown, Jonathan Ko, Lillian Y Chang, David D Wilkinson, Sean M Bidic, and Yoky Matsuoka. Mechanisms of the anatomically correct testbed hand. IEEE/ASME Transactions on Mechatronics, 18(1):238250, 2013.

[11] Thomas Feix, Roland Pawlik, Heinz-Bodo Schmiedmayer, Javier Romero, and Danica Kragic. A comprehensive grasp taxonomy. In Robotics, Science and Systems: Workshop on Understanding the Human Hand for Advancing Robotic Manipulation, pages 2-3, 2009.

[12] Clément Gosselin, Frédéric Pelletier, and Thierry Laliberté. An anthropomorphic underactuated robotic hand with 15 dofs and a single actuator. In IEEE International Conference on Robotics and Automation (ICRA), pages 749-754, 2008.
[13] Henry Gray. Anatomy of the human body. Lea \& Febiger, 1918.

[14] Giorgio Grioli, Manuel Catalano, Emanuele Silvestro, Simone Tono, and Antonio Bicchi. Adaptive synergies: an approach to the design of under-actuated robotic hands. In IEEE/RSJ International Conference on Intelligent Robots and Systems (IROS), pages 1251-1256, 2012.

[15] Shigeo Hirose and Yoji Umetani. The development of soft gripper for the versatile robot hand. Mechanism and Machine Theory, 13(3):351-359, 1978.

[16] Mary C Hume, Harris Gellman, Harry McKellop, and Robert H Brumfield. Functional range of motion of the joints of the hand. The Journal of Hand Surgery, 15(2): 240-243, 1990.

[17] Peter J Kyberd, Colin Light, Paul H Chappell, Jim M Nightingale, Dave Whatley, and Mervyn Evans. The design of anthropomorphic prosthetic hands: A study of the Southampton Hand. Robotica, 19(06):593-600, 2001.

[18] Thierry Laliberté, Lionel Birglen, and Clément Gosselin. Underactuation in robotic grasping hands. Machine Intelligence \& Robotic Control, 4(3):1-11, 2002.

[19] Raymond R Ma, Lael U Odhner, and Aaron M Dollar. A modular, open-source 3D printed underactuated hand. In IEEE International Conference on Robotics and Automation (ICRA), pages 2737-2743, 2013.

[20] Bruno Massa, Stefano Roccella, Maria Chiara Carrozza, and Paolo Dario. Design and development of an underactuated prosthetic hand. In IEEE International Conference on Robotics and Automation (ICRA), volume 4, pages 3374-3379, 2002.

[21] E. Spellerberg. Improvement in artificial arms, November 28 1865. URL https://www.google.ca/patents/ US51238. US Patent 51,238.

[22] N.T. Ulrich. Methods and apparatus for mechanically intelligent grasping, March 26 1996. URL https://www. google.com/patents/US5501498. US Patent 5,501,498. 